\title{
Edge helicons and repulsion of fundamental edge magnetoplasmons in the quantum Hall regime
}

\author{
O. G. Balev ${ }^{1,2}$, P. Vasilopoulos ${ }^{3}$, and Nelson Studart ${ }^{1}$
}

\author{
${ }^{1}$ Departmento de Fisica, Universidade Federal de São Carlos, 13565-905 São Carlos, \\ São Paulo, Brazil \\ ${ }^{2}$ Institute of Physics of Semiconductors, National Academy of Sciences, 45 Prospekt \\ Nauky, Kiev 252650, Ukraine \\ ${ }^{3}$ Concordia University, Department of Physics, 1455 de Maisonneuve Blvd O, Montréal, \\ Québec, Canada, H3G 1M8
}

\begin{abstract}
A quasi-microscopic treatment of edge magnetoplasmons (EMP) is presented for very low temperatures and confining potentials smooth on the scale of the magnetic length $\ell_{0}$ but sufficiently steep at the edges such that Landau level (LL) flattening can be discarded. The profile of the unperturbed electron density is sharp and the dissipation taken into account comes only from electron intra-edge and intra-LL transitions due to scattering by acoustic phonons. For wide channels and filling factors $\nu=1$ and 2 , there exist independent EMP modes spatially symmetric and antisymmetric with respect to the edge. Some of these modes, named edge helicons, can propagate nearly undamped even when the dissipation is strong. Their density profile changes qualitatively during propagation and is given by a rotation of a complex vector function. For $\nu>2$, the Coulomb coupling between the LLs leads to a repulsion of the uncoupled fundamental LL modes: the new modes have very different group velocities and are nearly undamped. The theory accounts well for the experimentally observed plateau structure of the delay times as well as for the EMP's period and decay rates.

PACS 73.20.Dx, 73.40.Hm
\end{abstract}

\section{INTRODUCTION}

Previous theoretical studies of edge magnetoplasmons (EMP), the low-frequency collective excitations which propagate along the edges of a two-dimensional electron gas (2DEG) subject to a normal magnetic field $B$, point out some important characteristics of EMP, e.g., the gapless excitation spectrum and the acoustic EMP. However, the authors of Refs. [1] and [2] have assumed density profiles which are infinitely sharp or smooth respectively and independent of the filling factor $\nu=n_{0} h /|e| B$, where $n_{0}$ is the electron density in the bulk of 2DEG. As a consequence, they do not reflect the Landau-level (LL) structure, as one can see in Fig. 1, in which we compare our calculated density profile for the cases of one and two occupied LLs, by assuming a smooth parabolic confining potential at the edge, with the results of Refs. [1] and [2]. This inadequacy is clearly manifested in the observed 5 plateau structure of the transit times reflecting that of the quantum Hall effect (QHE) plateaus and not accounted for in Ref. [2]. In addition, for a spatially homogeneous dissipation within the channel, the EMP damping was found quantized and independent of temperature 1$]$ or it was treated phenomenologically. [․ The calculated damping rates were 
strongly overestimated 3. Other drawbacks and the limited validity of the treatment of Ref. [1] in the QHE regime were pointed out in Refs. [4]- [6].

In this paper, we present a theory of EMP for integer $\nu$ in which the LL structure is taken into account. In doing so, we have assumed that the confining potential is sufficiently

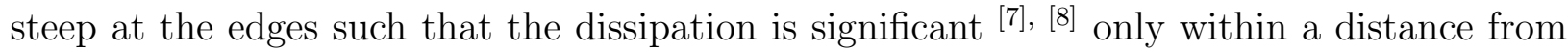
the edge of the order of the magnetic length $\ell_{0}=\sqrt{\hbar /|e| B}$ and that LL flattening [9] can be neglected. [10], 11] We show that, for $\nu>2$, the Coulomb coupling, between the charge excitations at the edges of different occupied LLs leads to a repulsion of the uncoupled fundamental LL modes. The new modes, which are nearly undamped, have very different group velocities.

For the 2D system with a vertical conductivity drop at the boundaries, it has been shown that the dissipation determines significantly the dispersion relation and the spatial structure of the EMP even in the regime of the QHE. The properties of the EMP have been expressed in terms of the components of the magnetoconductivity tensor of an infinite 2D system. Moreover, due to the very low frequency $\omega$ of the EMP, the dispersion relation could be written in terms of the static magnetoconductivity tensor. However, in Refs. [0] and [8] we have shown that, for a sufficiently smooth lateral confinement and in the QHE regime, the dissipation appears dominantly due to intralevel-intraedge transitions of electrons interacting with acoustic phonons and occurs mainly near the edges. For instance, in GaAs-based samples, piezoelectrical (PA) phonons are typically involved. In the linear response regime this is the main cause for dissipation in channels of width $W \lesssim 100 \mu \mathrm{m}$ and temperatures $T \lesssim 1 \mathrm{~K}$ if the group velocity of the edge states $v_{g}$ is larger than the speed of sound $s$. As in the case of dissipation in the bulk, it is exponentially suppressed as the temperature goes to zero. Under this circumstance, and the fact that the dissipation in the VolkovMikhailov model is homogeneous over the channel width, the previous results of Balev and Vasilopoulos 12], 13 are understood as a demonstration that the EMP properties reported in Ref. [1] can be strongly modified by dissipative processes localized near the channel edges. Here we assume very low temperatures which implies that the condition $k_{B} T \ll \hbar v_{g} / \ell_{0}$ is fulfilled. A brief account of some results of the present investigation has appeared in Ref. [13].

The organization of the paper is as follows. In Sec. II, by starting with the expressions for the inhomogeneous current densities and conductivities, we derive the integral equation for EMPs and present the general method for solving it. In Sec. III we calculate the dispersion relations and the spatial structure of the new edge waves at very low temperatures. Finally, in Sec. IV we compare our theory with experiment and present our concluding remarks.

\section{BASIC RELATIONS}

\section{A. Inhomogeneous current density in the quasi-static regime}

We consider a 2DEG confined to a strip in the $x-y$ plane with a width $W$ in the $y$ axis and length $L$ along a channel in the $x$ direction, under a strong magnetic field $B$ parallel to the $z$ axis. For simplicity, we consider the confining potential as parabolic at the edges such that $V_{y}=0$, for $y_{l}<y<y_{r}, V_{y}=m^{*} \Omega^{2}\left(y-y_{r}\right)^{2} / 2$ for $y>y_{r}>0$, and $V_{y}=m^{*} \Omega^{2}\left(y-y_{l}\right)^{2} / 2$ 
for $y<y_{l}<0$, where $y_{r, l}$ delimited the right and left edges of the flat part of $V_{y}$. We assume the condition $\left|k_{x}\right| W \gg 1$, so it is possible to consider an EMP along the right edge of the channel of the form $A\left(\omega, k_{x}, y\right) \exp \left[-i\left(\omega t-k_{x} x\right)\right]$ totally independent of the left edge. We consider only the linear response regime. For definiteness, we take the background dielectric constant $\epsilon$ to be spatially homogeneous. In the QHE regime and $\nu$ even, we neglect the Zeeman spin splitting. As for the case $\nu=1$, we assume that the spin splitting, caused by many-body effects, is strong enough to neglect the contribution related to the upper spinsplit LL. We also assume a smooth lateral confinement on the scale of the magnetic length $\ell_{0}=\left(\hbar / m^{*} \omega_{c}\right)^{1 / 2}$ such that $\Omega \ll \omega_{c}$, where $\omega_{c}=|e| B / m^{*}$ is the cyclotron frequency.

The EMP is practically quasi-static and its wavelength $\lambda$ is much larger than $\ell_{0}$. As in Refs. [12] and [13], we write the components of the current density in the form

$$
\begin{gathered}
j_{y}(y)=\sigma_{y y}(y) E_{y}(y)+\sigma_{y x}^{0}(y) E_{x}(y), \\
j_{x}(y)=\sigma_{x x}(y) E_{x}(y)-\sigma_{y x}^{0}(y) E_{y}(y)+\sum_{j} v_{g j} \rho_{j}\left(\omega, k_{x}, y\right),
\end{gathered}
$$

where $\sigma_{\mu \gamma}(y)$ and $E_{\gamma}(y)$ are the components of the conductivity tensor and the electric field respectively. Here we have suppressed the exponential factor $\exp \left[-i\left(\omega t-k_{x} x\right)\right]$ common to all terms in Eqs. (11) and (2). It is understood that $E_{\gamma}(y)$ depends on $\omega$ and $k_{x}$. The term $v_{g j} \rho_{j}\left(\omega, k_{x}, y\right)$ represents an advection contribution caused by a charge distortion $\rho_{j}\left(\omega, k_{x}, y\right)$ localized near the edge $y_{r j}$ of the $j$-th LL. In Eqs. (11) and (2) the contributions to $j_{\mu}(y)$, which are proportional to $E_{\gamma}(y)$, are microscopically obtained when the electric field is smooth on the scale of $\ell_{0}$. Even though this is not well justified for both components proportional to $E_{x}(y)$ and $E_{y}(y)$, we approximate these contributions by those obtained when $E_{x}(y)$ and $E_{y}(y)$ are smooth on the scale of $\ell_{0}$. This approximation is equivalent to neglecting nonlocal contributions to $j_{\mu} \propto \int d y^{\prime} \sigma_{\mu \gamma}\left(y, y^{\prime}\right) E_{\gamma}\left(y^{\prime}\right)$. For weak dissipation, it can be justified within a treatment based on the random-phase-approximation (RPA) 14] that includes nonlocal effects and edge-state screening, e.g., for the fundamental EMPs at $\nu=2,4,6$. The Hall conductivity is 8

$$
\sigma_{y x}^{0}(y)=\frac{e^{2}}{2 \pi \hbar} \sum_{n=0} \int_{-\infty}^{\infty} d y_{0 \alpha} f_{\alpha} \Psi_{n}^{2}\left(y-y_{0 \alpha}\right),
$$

where $\alpha \equiv\left\{n, k_{x \alpha}\right\}, y_{0 \alpha}=\ell_{0}^{2} k_{x \alpha}, \Psi_{n}(y)$ is the harmonic oscillator function, and $f_{\alpha} \equiv$ $f_{n}\left(k_{x \alpha}\right)=1 /\left[1+\exp \left(\varepsilon_{\alpha}-\varepsilon_{F}\right) / k_{B} T\right]$ is the Fermi-Dirac function. $\varepsilon_{F}$ is the Fermi level measured from the bottom of the lowest electric subband; for even $\nu$ the right-hand side of Eq. (3) should be multiplied by 2, the spin degeneracy factor. We point out that, for $\nu=1$ and $T=0$ and near the right edge, we obtain $\sigma_{y x}^{0}(y)=\left(e^{2} / 4 \pi \hbar\right)\left[1+\Phi\left(y_{r e}-y\right)\right]$, where $\Phi(x)$ is the probability integral, $y_{r e}=\ell_{0}^{2} k_{r e}$, and $f_{0}\left(k_{r e}\right)=1 / 2$. Notice then that $\sigma_{y x}^{0}(y)$, near the edge, decreases on the scale of $\ell_{0}$ and behaves like the density profile depicted by the dashed curve of Fig. 1. Considering only the right edge and the flat part of the confining potential for $y_{l} \leq y_{0 \alpha} \leq y_{r}$, we obtain the energy levels $\varepsilon_{\alpha}=\hbar \omega_{c}(n+1 / 2)$ and for $y_{0 \alpha} \geq y_{r}$, the energy spectrum can be written as

$$
\varepsilon_{\alpha} \equiv \varepsilon_{n}\left(k_{x \alpha}\right)=\hbar \omega_{c}(n+1 / 2)+m^{*} \Omega^{2}\left(y_{0 \alpha}-y_{r}\right)^{2} / 2
$$


This results implies that $\varepsilon_{\bar{n}}\left(k_{x \alpha}\right)$, as a function of $y_{0 \alpha}$, is smooth on the scale of $\sqrt{(2 \bar{n}+1)} \ell_{0}$, where $\bar{n}$ is the principal quantum number of the highest occupied LL. The energy spectrum (ब) of the $n$-th LL allows us to evaluate the group velocity of the edge states as $v_{g n}=\partial \varepsilon_{n}\left(k_{r}+\right.$ $\left.k_{e}^{(n)}\right) / \hbar \partial k_{x}=\hbar \Omega^{2} k_{e}^{(n)} / m^{*} \omega_{c}^{2}$ with the characteristic wave vector $k_{e}^{(n)}=\left(\omega_{c} / \hbar \Omega\right) \sqrt{2 m^{*} \Delta_{F n}}$, where $\Delta_{F n}=\varepsilon_{F}-(n+1 / 2) \hbar \omega_{c}$. The edge of the $n$-th LL is denoted by $y_{r n}=y_{r}+\ell_{0}^{2} k_{e}^{(n)}=$ $\ell_{0}^{2} k_{r n}$, where $k_{r n}=k_{r}+k_{e}^{(n)}, k_{r}=y_{r} / \ell_{0}^{2}$ and $W=2 y_{r 0}$. We can also write $v_{g n}=E_{e n} / B$, where $E_{e n}=\Omega \sqrt{2 m^{*} \Delta_{F n}} /|e|$ is the electric field associated with the confining potential $V_{y}$ at $y_{r n}$.

We consider only the electron-phonon interaction and neglect that of electrons with impurities, since the former is the most essential for the assumed conditions 16 . Following Refs. [12 and [13, we approximate $\sigma_{x x}(y)$ by $\sigma_{y y}(y)=\sum_{n=0}^{\bar{n}} \sigma_{y y}^{(n)}(y)$. Furthermore, at very low $T$ where $\hbar v_{g n} \gg \ell_{0} k_{B} T$ with $v_{g n}>s$ is satisfied, the Eq. (16) of Ref. [B] gives $\sigma_{y y}^{(n)}(y)=$ $\tilde{\sigma}_{y y}^{(n)} \Psi_{n}^{2}\left(\bar{y}_{n}\right), \bar{y}_{n}=y-y_{r n}$. For PA phonons and $\nu=2,4$, we have $\tilde{\sigma}_{y y}^{(n)}=3 e^{2} \ell_{0}^{4} c^{\prime} k_{B}^{3} T^{3} / \pi^{2} \hbar^{6} v_{g n}^{4} s$ where $c^{\prime}$ is the electron-phonon coupling constant. Note then that $\sigma_{y y}(y)$ is exponentially localized within a distance $\lesssim \ell_{0}$ from the LL edge $y_{r n}$. Notice also that for $v_{g n} / s \gg 1$ such that $1 \gg k_{B} T \ell_{0} / \hbar v_{g n}>s / \sqrt{2} v_{g n}$ we have $\tilde{\sigma}_{y y}^{(n)}=\sqrt{2} e^{2} \ell_{0}^{3} c^{\prime} k_{B}^{2} T^{2} / \pi^{5 / 2} \hbar^{5} v_{g n}^{4}$. Furthermore, for all cases it is assumed that the strong magnetic field condition $\sigma_{y y}(y) /\left|\sigma_{y x}^{0}(y)\right| \ll 1$ is fulfilled.

\section{B. Integral equation for EMPs with dissipation at the edges}

Using Eqs. (11)-(3), the Poisson's equation, and the linearized continuity equation, we obtain the following integral equation for $\rho\left(\omega, k_{x}, y\right)$

$$
\begin{aligned}
&-i \sum_{n}\left(\omega-k_{x} v_{g n}\right) \rho_{n}\left(\omega, k_{x}, y\right)+\frac{2}{\epsilon}\left[k_{x}^{2} \sigma_{x x}(y)\right. \\
&\left.-i k_{x} \frac{d}{d y}\left[\sigma_{y x}^{0}(y)\right]-\sigma_{y y}(y) \frac{d^{2}}{d y^{2}}-\frac{d}{d y}\left[\sigma_{y y}(y)\right] \frac{d}{d y}\right] \\
& \times \int_{-\infty}^{\infty} d y^{\prime} K_{0}\left(\left|k_{x}\right|\left|y-y^{\prime}\right|\right) \rho\left(\omega, k_{x}, y^{\prime}\right)=0,
\end{aligned}
$$

where $K_{0}(x)$ is the modified Bessel function. For the dissipationless classical 2D electron liquid Eq. (5) becomes identical with Eq. (4) of Ref. [2]. Furthermore, if the conductivity components are independent of $y$, for $|y|<W / 2$, Eq. (5) assumes the form of Eq. (15) of Ref. [1]. In order to solve Eq. (5), we remark that, for $\hbar v_{g n} \gg \ell_{0} k_{B} T$, we have $d\left[\sigma_{y x}^{0}(y)\right] / d y \propto \sum_{n=0}^{\bar{n}} \Psi_{n}^{2}\left(\bar{y}_{n}\right)$, whose spatial behavior is similar to that of $\sigma_{y y}(y)$. Then Eq. (5) shows that $\rho_{n}\left(\omega, k_{x}, y\right)$ will be concentrated within a region of extent $\sim \ell_{0}$ around the edge of the $n$-th LL. Further, the integral can be evaluated under the assumption $k_{x} \ell_{0} \ll 1$ and using the approximation $K_{0}(|x|) \approx \ln (2 /|x|)-\gamma$, where $\gamma$ is the Euler constant. Assuming $\Delta y_{m-1, m}=y_{r m-1}-y_{r m} \gg \ell_{0}$, (see Fig. 1 for $\nu=4$ ), we can neglect the exponentially small overlap between $\rho_{m-1}\left(\omega, k_{x}, y\right)$ and $\rho_{m}\left(\omega, k_{x}, y\right)$, for $m \leq \bar{n}$. It is then natural to attempt the exact solution in the form

$$
\rho\left(\omega, k_{x}, y\right)=\sum_{n=0}^{\bar{n}} \rho_{n}\left(\omega, k_{x}, y\right)=\sum_{n=0}^{\bar{n}} \Psi_{n}^{2}\left(\bar{y}_{n}\right) \sum_{l=0}^{\infty} \rho_{n}^{(l)}\left(\omega, k_{x}\right) H_{l}\left(\bar{y}_{n} / \ell_{0}\right),
$$


by using the Hermite polynomials $H_{l}(x)$ as a expansion basis. We call the terms $l=0, l=$ $1, l=2$, and so on, the monopole, dipole, quadrupole terms in the expansion of $\rho_{n}\left(\omega, k_{x}, y\right)$ relative to $y=y_{r n}$.

\section{EMPs for $\nu=2$ and $\nu=4$}

Below we first give the general formulas in the case $\nu=4(\bar{n}=1)$. ¿From them, we show how general formulas for $\nu=2(\bar{n}=0)$ follow up. For $\nu=4$ we multiply Eq. (5) by $H_{m}\left(\bar{y}_{0} / \ell_{0}\right)$ and integrate over $y$, from $y_{r 0}-\Delta y_{01} / 2$ to $y_{r 0}+\Delta y_{01} / 2$. Analogous integration, from $y_{r 1}-\Delta y_{01} / 2$ to $y_{r 1}+\Delta y_{01} / 2$, is repeated with $H_{m_{1}}\left(\bar{y}_{1} / \ell_{0}\right)$. With the abbreviations $\rho_{0}^{(m)}\left(\omega, k_{x}\right) \equiv \rho_{0}^{(m)}, a_{m k}\left(k_{x}\right) \equiv a_{m k}$, etc., we obtain, the following coupled systems of equations

$$
\begin{gathered}
\left(\omega-k_{x} v_{g 0}\right) \rho_{0}^{(m)}-\left(S_{0}+m S_{0}\right) \sum_{n=0}^{\infty} c_{m n} a_{m n} \rho_{0}^{(n)} \\
-\left(S_{0}+m S_{0}^{\varsigma}\right) \sum_{l=0}^{\infty} c_{m l} b_{m l} \rho_{1}^{(l)}=0 \\
\left(\omega-k_{x} v_{g 1}\right)\left[A_{m_{1}} \rho_{1}^{\left(m_{1}\right)}+B_{m_{1}} \rho_{1}^{\left(m_{1}+2\right)}+\rho_{1}^{\left(m_{1}-2\right)} / 2\right] \\
-\left(S_{1}+m_{1} S_{1}^{\varsigma}\right)\left[\sum_{n=0}^{\infty} c_{m_{1} n} b_{n m_{1}} \rho_{0}^{(n)}+\sum_{j=0}^{\infty} c_{m_{1} j} d_{m_{1} j} \rho_{1}^{(j)}\right] \\
+2 \sqrt{m_{1}} S_{1}\left[\sum_{n=0}^{\infty} c_{m_{1} n} \tilde{b}_{n, m_{1}} \rho_{0}^{(n)}+\sum_{j=0}^{\infty} c_{m_{1 j} j} \tilde{d}_{m_{1}, j} \rho_{1}^{(j)}\right]=0
\end{gathered}
$$

where

$$
\begin{gathered}
a_{m n}=a_{n m}=\int_{-\infty}^{\infty} d x \Psi_{m}(x) \Psi_{0}(x) \int_{-\infty}^{\infty} d x^{\prime} \\
\times K_{0}\left(\left|k_{x}\right|\left|x-x^{\prime}\right|\right) \Psi_{n}\left(x^{\prime}\right) \Psi_{0}\left(x^{\prime}\right), \\
b_{m n}=\int_{-\infty}^{\infty} d x \Psi_{m}(x) \Psi_{0}(x) \int_{-\infty}^{\infty} d x^{\prime} \\
\times 2\left(x^{\prime} / \ell_{0}\right)^{2} K_{0}\left(\left|k_{x}\right|\left|x-x^{\prime}+\Delta y_{01}\right|\right) \Psi_{n}\left(x^{\prime}\right) \Psi_{0}\left(x^{\prime}\right) .
\end{gathered}
$$

Other coefficients are given as

$$
\begin{aligned}
\tilde{b}_{m n}= & \int_{-\infty}^{\infty} d x \Psi_{m}(x) \Psi_{0}(x) \int_{-\infty}^{\infty} d x^{\prime} \\
& \times K_{0}\left(\left|k_{x}\right|\left|x-x^{\prime}+\Delta y_{01}\right|\right) \Psi_{n-1}\left(x^{\prime}\right) \Psi_{1}\left(x^{\prime}\right), \\
d_{m n}= & \left(2 / \ell_{0}^{2}\right) \int_{-\infty}^{\infty} d x \Psi_{m}(x) x \Psi_{1}(x) \int_{-\infty}^{\infty} d x^{\prime} \\
& \times K_{0}\left(\left|k_{x}\right|\left|x-x^{\prime}\right|\right) \Psi_{n}\left(x^{\prime}\right) x^{\prime} \Psi_{1}\left(x^{\prime}\right),
\end{aligned}
$$




$$
\begin{aligned}
\tilde{d}_{m n}= & \left(\sqrt{2} / \ell_{0}\right) \int_{-\infty}^{\infty} d x \Psi_{m-1}(x) \Psi_{1}(x) \int_{-\infty}^{\infty} d x^{\prime} \\
& \times K_{0}\left(\left|k_{x}\right|\left|x-x^{\prime}\right|\right) \Psi_{n}\left(x^{\prime}\right) x^{\prime} \Psi_{1}\left(x^{\prime}\right) .
\end{aligned}
$$

In addition, $c_{m n}=\sqrt{2^{n} n ! / 2^{m} m !}, A_{m_{1}}=\left(2 m_{1}+1\right), B_{m_{1}}=\left(m_{1}+2\right)\left(2 m_{1}+2\right), S_{j}=$ $2\left(k_{x} \tilde{\sigma}_{y x}^{0}-i k_{x}^{2} \tilde{\sigma}_{x x}^{(j)}\right) / \epsilon, S_{j}=-4 i \tilde{\sigma}_{y y}^{(j)} / \epsilon \ell_{0}^{2}$, and $\tilde{\sigma}_{y x}^{0}=e^{2} / \pi \hbar$. Notice that here in the interior part of the channel $\sigma_{y x}^{0}(y)=\sigma_{y x}^{0}=2 \tilde{\sigma}_{y x}^{0}=2 e^{2} / \pi \hbar$.

Furthermore, for $\nu=2(\bar{n}=0)$ the third term in Eqs. (7) and (8) is absent and we obtain explicitly

$$
\left(\omega-k_{x} v_{g 0}\right) \rho_{0}^{(m)}-\left(S_{0}+m S_{0}^{\iota}\right) \sum_{n=0}^{\infty} c_{m n} a_{m n} \rho_{0}^{(n)}=0
$$

In this case we obtain $\sigma_{y x}^{0}(y)=\sigma_{y x}^{0}=\tilde{\sigma}_{y x}^{0}=e^{2} / \pi \hbar$ in the inner part of the channel.

\section{EDGE WAVES}

\section{A. Edge modes for $\nu=2(1)$}

In what follows we assume $\nu=2$ but the results obtained can be easily extended to the case of $\nu=1$, when only the lowest spin-split LL is occupied. Equations (6) and (14) show that there exist independent modes, spatially symmetric, $\rho^{s}\left(\omega, k_{x}, y\right)$, or antisymmetric, $\rho^{a}\left(\omega, k_{x}, y\right)$, with respect to $y=y_{r 0}$ (see also Ref. [12]). They correspond to $l$ even or odd, respectively, in Eq. (6). Notice that in this case $\bar{n}=0$ and all edge modes belong to the $n=0$ LL. However, within our quasi-microscopic approach they typically include mixing of the $n=0$ LL with some higher empty LLs.

We first consider the lowest antisymmetric mode for weak dissipation [12] $\eta=$ $\tilde{\sigma}_{y y}^{(0)} /\left(\ell_{0}^{2} \sigma_{y x}^{0}\left|k_{x}\right|\right) \ll 1 / 4$. As we neglect its coupling with higher antisymmetric modes, it becomes purely dipole and corresponds to $l=1$ and $n=0$ in Eq. (6). To take into account the effect of inter-mode coupling on this pure dipole $(l=1)$ mode we will neglect damping. Then, as shown in Ref. [12], the pure dipole mode has a dimensionless velocity $v_{d i p}=\left(\omega / k_{x}-v_{g o}\right) /\left(2 \sigma_{y x}^{0} / \epsilon\right)$ given by $v_{d i p}=a_{11} \approx 0.4996$. Furthermore, if only the interaction of this mode with the octupole mode is considered, the velocity of the resulting renormalized dipole mode becomes $v_{\text {dip }}=\left(a_{11}+a_{33}+\sqrt{\left(a_{11}-a_{33}\right)^{2}+4 a_{13}^{2}}\right) / 2 \approx 0.5963$. Here only the $l=1$ and $l=3$ terms in Eq. (6) are involved. It is seen that due to the interaction with the $l=3$ mode, $v_{\text {dip }}$ becomes $\approx 20 \%$ higher. To take into account the interaction of the dipole mode with the $l=3$ and $l=5$ modes, we retain the terms $l=1,3$ and $l=5$ in Eq. (6). From Eq. (14), for $m=1,3$ and $m=5$, we obtain a system of three linear equations for $\rho_{0}^{(1)}, \rho_{0}^{(3)}$, and $\rho_{0}^{(5)}$ that leads to $v_{\text {dip }} \approx 0.6287$. Thus, the interaction with the $l=5$ mode led to a $v_{d i p}$ only $\approx 5 \%$ higher than that where only the terms with $l=1,3$ were retained. Hence $v_{d i p}$, and consequently the dispersion relation of the dipole mode, exhibits fast convergence when more $l$ terms are taken into account. Notice that, within the present quasi-microscopic approach, the dipole mode has a purely acoustic dispersion relation. The charge-density profile $\delta \rho(y)$ of the dipole mode is shown in Fig. 2 where we plot $\delta \rho(y) \equiv \tilde{\rho}\left(v_{d i p}, y\right)=\sqrt{\pi} \ell_{0} \rho\left(\omega_{\text {dip }}\left(k_{x}\right), k_{x}, y\right) / \rho^{(1)}\left(\omega_{\text {dip }}\left(k_{x}\right), k_{x}\right)$ as a function of $\bar{y}_{0} / \ell_{0}$. The 
dashed, short-dashed, and solid curves are obtained, respectively, with one $(l=1)$, two $(l=1,3)$ or three $(l=1,3,5)$ terms retained in the expansion (6). The profile shows a clear convergence already for $l \leq 5$.

For very strong dissipation $\eta \gg K$, where $K=\ln \left(1 /\left|k_{x} \ell_{0}\right|\right)+1 / 2$, we consider only the weakly damped EMP branch that was termed low-frequency edge helicon (LFEH) in Refs. [12] and [13]. This mode is symmetric and its real part $\operatorname{Re} \omega\left(k_{x}\right) \equiv \operatorname{Re} \omega_{E H}^{L F} \approx$ $k_{x} v_{g 0}+\left(2 \sigma_{y x}^{0} k_{x} / \epsilon\right)(K-1 / 4)$ is very close to Re $\omega\left(k_{x}\right)$ of the fundamental EMP of the $n=0 \mathrm{LL}$, namely, $\operatorname{Re} \omega_{E H}^{(0)} \approx k_{x} v_{g 0}+\left(2 \sigma_{y x}^{0} k_{x} / \epsilon\right)(K+1 / 4)$. The fundamental EMP of the $n=0$ LL has a dispersion $\omega_{E H}^{(0)}=k_{x} v_{g 0}+S_{0}(K+1 / 4)+S_{0} / 4 K$ for both weak dissipation, $\eta \ll 1 / 4$, and strong dissipation, $K \gg \eta \gg 1 / 4$. [cf. Refs. [12] and [13]]. In the latter case the fundamental EMP was termed [12], [13] high-frequency edge helicon (HFEH). In both regimes of dissipation, the fundamental EMP of $n=0$ LL has mainly a monopole character 12], [13]: in the first case its profile satisfies $\left|\rho_{0}^{(2)} / \rho_{0}^{(0)}\right| \approx(1 / 8 K) \ll 1$, and in the second, $\left|\rho_{0}^{(2)} / \rho_{0}^{(0)}\right| \approx(\eta / 2 K) \ll 1$. In either case the fact of considering only two terms in Eq. (6),$l=0$ and $l=2$, is well justified. The latter is not the case for LFEH as shown in Ref. [13].

To support the statements made above we present in Figs. 3 and 4 the evolution of the dimensionless charge density profile of the LFEH given by $\delta \rho_{r}=\sqrt{\pi} \ell_{0} \operatorname{Re}$ $\left[\rho\left(\omega, k_{x}, y\right) / \rho_{0}^{(0)}\left(\omega, k_{x}\right)\right]$ and $\delta \rho_{i}=\sqrt{\pi} \ell_{0} \operatorname{Im}\left[\rho\left(\omega, k_{x}, y\right) / \rho_{0}^{(0)}\left(\omega, k_{x}\right)\right]$, respectively, as one increases the number of terms considered in the expansion for $K / \eta=0.01$. Notice that as $\delta \rho_{r}$ represents the profile for a particular phase of the wave, then $\delta \rho_{i}$ represents it for a phase shifted by $\pm \pi / 2$. Since $\delta \rho(y)$ is symmetric with respect to the edge, only the half part of the profile is shown in Figs. 3 and 4. In Fig. 3, curve 1 represents $\delta \rho_{r}$ when only the $l=0$ term is retained in the expansion and curve 2 shows $\delta \rho_{r} \approx \sqrt{\pi} \ell_{0}\left[\Psi_{0}^{2}\left(\bar{y}_{0}\right)+\sqrt{2} \Psi_{2}\left(\bar{y}_{0}\right) \Psi_{0}\left(\bar{y}_{0}\right)\right]$ when two terms $l=0,2$ are retained. As these curves are essentially different, more $l$ terms should be considered in Eq. (6). Thus, to describe better the profile $\delta \rho_{r}$ we also plot curves 3,4 , and 5 obtained, respectively, when 3 to 5 even $l$ terms are retained in Eq. (6). This leads to systems of 3, 4, and 5 equations following from Eq. (14). For instance, the curve 5 was obtained by retaining the terms $m=0,2,4,6$, and 8 . As it is seen in Fig. 3, keeping 4 or 5 terms in the $l$ summation leads already to a rapid convergent form of $\delta \rho_{r}$ without altering much its oscillatory character or changing its magnitude. In Fig. 4, similar results are depicted for the profile $\delta \rho_{i}$ when 2,3, 4, and 5 even $l$ terms are retained in Eq. (6). Notice that the contribution related to the monopole term, $l=0$, is absent; since the total edge charge $\int d y \delta \rho_{i}=0$. Therefore $\delta \rho_{i}$ shows essentially a stronger oscillatory behavior than $\delta \rho_{r}$ and correspondingly a slower convergence. However, curves 4 and 5 have approximately the same magnitude in the region where $\left|\bar{y}_{0}\right| / \ell_{0} \leq 1.5$. Moreover, for $\left|\bar{y}_{0}\right| / \ell_{0} \geq 1.5$ these curves exhibit both same spatial behavior and magnitude.

In Figs. 5 and 6, we plot the same profiles as in Figs. 3 and 4 , respectively, for $K / \eta=0.1$. Curves 1 and 2 in Fig. 5 shows $\delta \rho_{r}$ with, respectively, only one $(l=0)$ or two $(l=0,2)$ terms retained in Eq. (6). As can be seen, keeping 3,4 or 5 terms in the $l$ summation leads already to a clear convergence in the form of $\delta \rho_{r}$. Figure 6 shows the profile $\delta \rho_{i}$ and the curves are marked as in Fig. 4. Because the contribution of the monopole term is absent, $\delta \rho_{i}$ has an essential oscillatory behavior that is rather similar for curves 4 and 5 as the spatial positions of the extrema for both curves almost coincide. The dispersion relation of the LFEH obtained for cases represented by curve 2 in Figs. 3-6 is given by 12, 13. 


$$
\omega_{E H}^{L F}=k_{x} v_{g 0}+\left\{\left(2 \sigma_{y x}^{0} k_{x} / \epsilon\right)-\left[i \tilde{\sigma}_{y y}^{(0)} / \eta^{2} \ell_{0}^{2} \epsilon\right]\right\}(K-1 / 4) .
$$

When more $l$ terms are taken into account only the imaginary part $\operatorname{Im} \omega_{E H}^{L F}$ changes significantly. For instance, with $2,3,4$, or 5 terms retained and $K / \eta=0.01$, the dispersion relations, in Figs. 3 and 4, are given, respectively, by $\Omega_{E H}^{L F}=\omega_{E H}^{L F} / S_{0} \approx[(K-0.25)-0.005 i]$, $\Omega_{E H}^{L F} \approx[(K-0.50)-0.062 i], \Omega_{E H}^{L F} \approx[(K-0.63)-0.117 i]$, and $\Omega_{E H}^{L F} \approx[(K-0.57)-0.133 i]$. It is clearly seen that with 4 and 5 even terms taken into account $\operatorname{Im} \omega_{E H}^{L F}$ shows rapid convergence to its exact value. Despite the very strong dissipation the LFEH is very weakly damped since Re $\left|\omega_{E H}^{L F}\right| \gg \operatorname{Im}\left|\omega_{E H}^{L F}\right|$. Furthermore, in contrast with Ref. [1], the real part Re $\omega_{E H}^{L F}$ is independent of $T$ and the damping rate $\operatorname{Im} \omega_{E H}^{L F}$ is not quantized but varies as $T^{-3}$ or $T^{-2}$.

Due to specific properties of LFEH, such as, for instance, the essential charge oscillations transversal to the edge, we may distinguish the LFEH from the fundamental EMP of the $n=0$ LL. For strong dissipation the latter mode is also called the HFEH of the $n=0 \mathrm{LL}$. It is worth notice that for the fundamental mode we have $2\left(K k_{x} \ell_{0}\right)^{2} \ll 1$ due to the long wavelength condition $k_{x} \ell_{0} \ll 1$. Therefore $S_{j}$ can be well approximated by its real part for the mode.

\section{B. Repulsion of fundamental EMPs for $\nu=4$}

Although here the condition $\Delta y_{01} / \ell_{0} \gg 1$ is well justified, as can be seen in Fig. 1, and simplifies the treatment, the system of Eqs. (7) and (8) can be strongly coupled by long-range Coulomb interaction between the edges of the LL. If we neglect this inter-edge Coulomb coupling in Eqs. (77) and (8), by setting the coefficients $b_{m n}$ and $\tilde{b}_{m n}$ equal to zero, then Eq. (7) leads to Eq. (14) for the $\nu=2$ case and all edge modes of the $n=1$ LL are decoupled from those of the $n=0 \mathrm{LL}$.

Assuming the latter, we first consider the symmetric edge modes of the $n=1$ LL. We consider both cases of strong dissipation and weak dissipation. In the former case the fundamental mode of $n=1 \mathrm{LL}$ can be called the HFEH of the $n=1 \mathrm{LL}$. To treat the fundamental mode of $n=1$ LL properly it is sufficient to consider the first two even terms in the sum over $n$ in Eq. (61), namely $\rho_{1}^{(0)}$ and $\rho_{1}^{(2)}$. Then from Eq. (8) for $m_{1}=0$ and $m_{1}=2$ we obtain a system of two linear equations for $\rho_{1}^{(0)}, \rho_{1}^{(2)}$. The corresponding dispersion relations of the HFEH of the $n=1$ LL becomes $\omega_{E H}^{(1)} \approx k_{x} v_{g 1}+S_{1}(K-1 / 4)+S_{1}^{*} / 12 K$. The other branch has the dispersion $\omega_{3}^{(1)} \approx k_{x} v_{g 1}+\left(S_{1}+2 S_{1}^{c}\right) / 4$. In analogy with the fundamental EMP of the $n=0 \mathrm{LL}$, the $n=1 \mathrm{LL}$ fundamental EMP, decoupled from the excitations of the $n=0 \mathrm{LL}$, is very weakly damped even for strong dissipation. Now if we omit the term of $\rho_{1}^{(2)}$ in Eq. (6), i.e., if we neglect the interaction between the monopole and quadrupole excitations of the $n=1 \mathrm{LL}$, the decoupled fundamental mode of $n=1 \mathrm{LL}$ has a dispersion relation given by $\omega_{E H}^{(1)}$ without the damping term. This holds for the $n=0 \mathrm{LL}$ as well, i.e., for its purely monopole excitation $\rho_{0}^{(0)}$ with dispersion given by $\omega_{E H}^{(0)}$ without the damping term.

If we take into account the Coulomb coupling between the pure monopole modes $\rho_{0}^{(0)}$ and $\rho_{1}^{(0)}$, their dispersions change drastically. For $\left|k_{x}\right| \Delta y_{01} \ll 1$, the dispersion of the renormalized fundamental mode of the $n=0$ LL becomes $\omega_{+}^{(01)} \approx k_{x}\left(v_{g 0}+v_{g 1}\right) / 2+$ 
$(2 / \epsilon) k_{x} \tilde{\sigma}_{y x}^{0}\left[2 \ln \left(1 / k_{x} \ell_{0}\right)-\ln \left(\Delta y_{01} / \ell_{0}\right)+3 / 5\right]$ and that of the $n=1 \mathrm{LL} \omega_{-}^{(01)} \approx k_{x}\left(v_{g 0}+\right.$ $\left.v_{g 1}\right) / 2+(2 / \epsilon) k_{x} \tilde{\sigma}_{y x}^{0}\left[\ln \left(\Delta y_{01} / \ell_{0}\right)+2 / 5\right]$. The dispersion relation $\omega_{+}^{(01)}\left(k_{x}\right)$ is similar to that of the fundamental $j=0$ mode of Ref. [2] and to the EMP of Ref. [1] since all of them have the term $\propto k_{x} \ln \left(1 / k_{x}\right)$. Notice that $\omega_{+}^{(01)}\left(k_{x}\right)$ is essentially different from the frequency of the decoupled $n=0$ LL fundamental mode $\omega \approx k_{x} v_{g 0}+(2 / \epsilon) \tilde{\sigma}_{y x}^{0} k_{x}\left[\ln \left(1 / k_{x} \ell_{0}\right)+3 / 4\right]$. In contrast with $\omega_{+}^{(01)}\left(k_{x}\right)$ the dispersion of the renormalized fundamental EMP of the $n=1 \mathrm{LL}$, $\omega_{-}^{(01)}\left(k_{x}\right)$, becomes purely acoustic. Its phase velocity is larger than that of the $j=1$ mode of Ref. [2] for $\Delta y_{01} / \ell_{0} \geq 5$. Here we observe that the term related to the edge velocities, $k_{x}\left(v_{g 0}+v_{g 1}\right) / 2$, is typically much smaller than the second term related to the electronelectron interaction. The spatial dependence of $\rho\left(\omega, k_{x}, y\right)$ for the renormalized fundamental mode of the $n=0 \mathrm{LL}$ is approximately $\propto\left[\Psi_{0}^{2}\left(\bar{y}_{0}\right)+\Psi_{1}^{2}\left(\bar{y}_{1}\right)\right]$ and that for the renormalized fundamental mode of the $n=1 \mathrm{LL}$ is $\propto\left[\Psi_{0}^{2}\left(\bar{y}_{0}\right)-\Psi_{1}^{2}\left(\bar{y}_{1}\right)\right]$.

The above results for Re $\omega\left(k_{x}\right)$ of the coupled fundamental EMP of the $n=0$ and $n=1$ LL's remain practically unchanged if more terms $\rho_{0}^{(j)}$ and $\rho_{1}^{(j)}$, for $j \geq 1$, are retained. Notice that, due to the presence of the inter-edge Coulomb coupling, the symmetry of the problem with respect to $y_{r 0}$ or $y_{r 1}$ is absent and hence, in principle, $\rho_{0}^{(j)}$ and $\rho_{1}^{(j)}$ with $j$ even and odd, must be included. Nevertheless, taking into account such additional terms leads to essential contributions to the damping rates of the renormalized fundamental EMPs of the $n=0$ and $n=1$ LLs. From $\Delta y_{01} / \ell_{0} \gg 1, k_{x} \Delta y_{01} \ll 1$ and the properties of the coefficients $a_{m n}$, $b_{m n}$, etc., we find that the most important terms in the sums of Eq. (6) are $\rho_{0}^{(0)}, \rho_{0}^{(2)}, \rho_{1}^{(0)}$, and $\rho_{1}^{(2)}$. Calculations show that the odd terms here are negligibly small. Dropping the less important term $\rho_{0}^{(2)}$, this leads to a system of three coupled equations giving three branches $\tilde{\omega}_{ \pm}^{(01)}$ and $\omega_{3}^{(01)}$. The dispersion relation of the renormalized fundamental EMP of the $n=0$ $\mathrm{LL}$ is given by

$$
\tilde{\omega}_{+}^{(01)}=k_{x}\left(v_{g 0}+v_{g 1}\right) / 2+(2 / \epsilon) k_{x} \tilde{\sigma}_{y x}^{0}\left[2 \ln \left(1 / k_{x} \ell_{0}\right)-\ln \left(\Delta y_{01} / \ell_{0}\right)+3 / 5\right]+S_{1}^{\varsigma} / 16 K
$$

and that of the $n=1$ LL by

$$
\tilde{\omega}_{-}^{(01)}=k_{x}\left(v_{g 0}+v_{g 1}\right) / 2+(2 / \epsilon) k_{x} \tilde{\sigma}_{y x}^{0}\left[\ln \left(\Delta y_{01} / \ell_{0}\right)+2 / 5\right]+S_{1}^{c} /\left\{24\left[\ln \left(\Delta y_{01} / \ell_{0}\right)+\gamma+1 / 4\right]\right\} .
$$

The coupled fundamental EMPs $\omega_{ \pm}^{(01)}$ are very weakly damped. Notice that the neglect of the $\rho_{0}^{(2)}$ term in the calculation of the damping rates is justified for substantially greater dissipation at the edge of the $n=1 \mathrm{LL}$ than that at that of the $n=0 \mathrm{LL}$. Indeed, $S_{j} \propto v_{g j}^{-4}$ and the group velocity $v_{g 0}$ is typically substantially larger than $v_{g 1}$. So if we neglect the inter-edge Coulomb coupling, the damping rate of the $\omega_{E H}^{(1)}$ branch is three times larger than that of the $\omega_{E H}^{(0)}$ branch for $v_{g 0} / v_{g 1}=\sqrt{3}$.

\section{DISCUSSION AND CONCLUDING REMARKS}

We have introduced a realistic model for the confining potential $V_{y}$ and took it sufficiently steep at the edge that LL flattening can be discarded 116]. For $\nu=2$, 4, we have neglected the spin splitting. This is a reasonable approximation in the bulk of the channel 
but its validity near the edges is not clear in view of the work of Refs. [15] and [16. We now compare our results presented in Sec. III A with the experimental ones by Ashoori et al. [6] for $\nu=1, T=0.3 \mathrm{~K}$, and $B=5.1 \mathrm{~T}$. Using the experimental value $\Omega=7.8 \times 10^{11} / \mathrm{sec}$, we obtain $v_{g 0}=8.8 \times 10^{5} \mathrm{~cm} / \mathrm{sec}$. This leads to $\tilde{\sigma}_{y y}^{(0)} \propto T^{3}$. By using a typical excited wave vector $q \simeq \pi / 2 L_{p}$, where $L_{p}=10 \mu \mathrm{m}$ is the side of the square pulse, we find that all modes presented in that section are very strongly damped except the fundamental mode $\omega_{E H}^{(0)}$ which is very weakly damped. Its damping rate is $\operatorname{Im} \omega_{E H}^{(0)} \approx 2 \times 10^{7} / \mathrm{sec}$ and its period of travel $T_{t r} \approx 3.4 \times 10^{-9} \mathrm{sec}$, in agreement with the experimental value. Here in the manner of Ref. [12 we have taken into account that air is close to the 2DEG in the experiment

The dispersion relations given by Eqs. (16) and (17), for $\nu=4$, correspond to the experimental parameters $B=2.06 \mathrm{~T}$ and $T=1.5 \mathrm{~K}$ of Ref. [3]. Using the experimentally determined 17] $\Omega=7.8 \times 10^{11} / \mathrm{sec}$ gives $\Omega / \omega_{c} \approx 0.14, \Delta y_{01} / \ell_{0} \approx 6, v_{g 0}=2.3 \times 10^{6} / \mathrm{sec}$, and $v_{g 0} / v_{g 1}=\sqrt{3}$. The spectrum of the $\nu=4$ modes, shown in Fig. (3a) of Ref. [3], is very well described by the dispersion of the renormalized fundamental modes given by Eqs. (16) and (17). The same holds for the $\nu=4$ mode of Fig. 3 (b) of Ref. [3]. The mode $\omega_{3}^{(01)}$ is strongly damped. Taking $\epsilon=6.75$, its decay rate is $\operatorname{Im} S_{1}^{c} / 2 \approx 2 \tilde{\sigma}_{y y}^{(1)} / \epsilon \ell_{0}^{2} \approx 1.3 \times 10^{10} /$ sec. The latter is still smaller than that of the $j=1$ branch of Ref. [2], $1 / \tau_{1} \approx 2 \times 10^{10} / \mathrm{sec}$, which becomes four times larger for $B=1 \mathrm{~T}$, due to the $B^{-2}$ behavior. The decay rate of the $j=0$ mode of Ref. [2] is $1 / \tau_{0} \approx 1.7 \times 10^{9} / \mathrm{sec}$ whereas that of the $\tilde{\omega}_{+}^{(01)}$ mode, given by Eq. (16), is about ten times smaller, $\operatorname{Im} \tilde{\omega}_{+}^{(01)} \approx 2.1 \times 10^{8} / \mathrm{sec}$. Moreover, the damping rate of the $\tilde{\omega}_{-}^{(01)}$ mode, given by Eq. (17), is much less than that of the $j=1$ mode of Ref. [2], since we have $\operatorname{Im} \tilde{\omega}_{-}^{(01)} \approx 5.6 \times 10^{8} / \mathrm{sec} \ll 1 / \tau_{1} \approx 2 \times 10^{10} / \mathrm{sec}$. Thus, the decay rates of the $\tilde{\omega}_{ \pm}^{(01)}$ modes should be much closer to those of the experiment [3] than the previous strongly overestimated ones 2 . With regard to the delay times $t_{d}$ for the sample with length $L_{x}=320 \mu \mathrm{m}$, we obtain $t_{d}=1.2 \times 10^{-10}$ sec for the $\tilde{\omega}_{+}^{(01)}$ mode and $t_{d}=6.9 \times 10^{-10}$ sec for the $\tilde{\omega}_{-}^{(01)}$ mode, in very good agreement with the experimental data 3 . From the previous discussion, it follows that the slower mode observed [3] for $\nu=4$ is not the $j=1$ mode of Ref. [2] but the present $\tilde{\omega}_{-}^{(01)}$ mode, i.e., the renormalized fundamental EMP of the $n=1$ LL. It is also clear that our theory accounts for the existence of the plateaus in $t_{d}$ as the quantized Hall conductivity appears naturally in all dispersion relations.

\section{ACKNOWLEDGMENTS}

This work was supported by Brazilian FAPESP Grants No.98/10192-2 and 95/0789-3, Canadian NSERC Grant No. OGP0121756. In addition, O. G. B. acknowledges partial support by the Ukrainian SFFI Grant No. 2.4/665, and N. S. is grateful do Brazilian CNPq for a research fellowship.

${ }^{2}$ E-mail: vbalev@power.ufscar.br

${ }^{3}$ E-mail: takis@boltzmann.concordia.ca

${ }^{1}$ E-mail: studart@power.ufscar.br 
[1] V. A. Volkov and S. A. Mikhailov, Zh. Eksp. Teor. Fiz. 94, 217 (1988) [Sov. Phys. JETP 67, 1639 (1988)] .

[2] I. L. Aleiner and L. I. Glazman, Phys. Rev. Lett. 72, 2935 (1994).

[3] G. Ernst, R. J. Haug, J. Kuhl, K. von Klitzing, and K. Eberl, Phys. Rev. Lett. 77, 4245 (1996).

[4] V. I. Tal'yanskii, M. Wassermeier, A. Wixforth, J. Oshinowo, J. P. Kotthaus, I. E. Batov, G. Weinmann, H. Nikel, and W. Schlapp, Surf, Sci. 229, 40 (1990).

[5] M. Wassermeier, J. Oshinowo, J. P. Kotthaus, A. H. MacDonald, C. T. Foxon, and J. J. Harris, Phys. Rev. B 41, 10287 (1990).

[6] R. C. Ashoori, H. L. Stormer, L. N. Pfeiffer, K. W. Baldwin, and K. West, Phys. Rev. B 45, 3894 (1992).

[7] O. G. Balev and P. Vasilopoulos,(a) Phys. Rev. B 47, 16410 (1993); (b) ibid. 50, 8706 (1994); (c) ibid. 50, 8727 (1994).

[8] O. G. Balev and P. Vasilopoulos, Phys. Rev. B 54, 4863 (1996).

[9] D. B. Chklovskii, B. I. Shklovskii, and L. I. Glazman, Phys. Rev. B 46, 4026 (1992).

[10] T. Suzuki and T. Ando, J. Phys. Soc. Jpn, 62, 2986 (1993).

[11] L. Brey, J. J. Palacios, and C. Tejedor, Phys. Rev. B 47, 13884 (1993).

[12] O. G. Balev and P. Vasilopoulos, Phys. Rev. B 56, 13252 (1997).

[13] O. G. Balev and P. Vasilopoulos, Phys. Rev. Lett. 81, 1481 (1998).

[14] O. G. Balev and P. Vasilopoulos, Phys. Rev. B, in press; O. G. Balev, P. Vasilopoulos, and N. Studart, Proc. XXIV International Conference on Physics of Semiconductors, Jerusalem, World Scientific (1999).

[15] J. Dempsey, B. Y. Gelfand, and B. I. Halperin, Phys. Rev. Lett. 70, 3639 (1993).

[16] O. G. Balev and P. Vasilopoulos, Phys. Rev. B 56, 6748 (1997).

[17] G. Muller, D. Weiss, A. V. Khaetskii, K. von Klitzing, S. Koch, H. Nickel, W. Schlapp, and R. Losch, Phys. Rev. B 45, 3932 (1992).

FIG. 1. Unperturbed electron density $n_{0}(y)$, normalized to the bulk value $n_{0}$, as a function of $y / \ell_{0}$. The thick solid curve is the model of Ref. 1 and the dotted curve that of Ref. 2 for $n_{0}(y) / n_{0}=(2 / \pi) \arctan \left[\left(y_{r e}-y\right) / a\right]^{1 / 2}, a / \ell_{0}=20$. The dashed and solid curves show the calculated profile for $\nu=1,2$ and for $\nu=4$, respectively. The star and open dots denote the edges of the $n=1$ and $n=0$ LL's.

FIG. 2. Dimensionless charge density profile $\tilde{\rho}(y) \equiv \rho_{\text {dip }}$ for a dipole mode as a function of $\bar{y}_{0} / \ell_{0}$; $\nu=2(1)$ and $\bar{n}=0$. The dashed, short-dashed, and solid curves correspond to one $(l=1)$, two $(l=1,3)$, and three $(l=1,3,5)$ terms, respectively, kept in the expansion (6).

FIG. 3. Dimensionless charge density profile $\rho_{r}(y)=\operatorname{Re} \delta \rho$ of the low-frequency edge helicon $(\mathrm{LFEH})$ as a function of $\bar{y}_{0} / \ell_{0}$ for $K / \eta=0.01 ; \nu=2(1), \bar{n}=0$. The number of even $l$ terms retained in Eq. (6) is shown next to the curves.

FIG. 4. Dimensionless charge density profile $\rho_{i}(y)=\operatorname{Im} \delta \rho$ of the low-frequency edge helicon (LFEH) as a function of $\bar{y}_{0} / \ell_{0}$ for $K / \eta=0.01 ; \nu=2(1), \bar{n}=0$. The number of even $l$ terms retained in Eq. (6) is shown next to the curves. 
FIG. 5. Same as in Fig. 3 for $K / \eta=0.1$.

FIG. 6. Same as in Fig. 4 for $K / \eta=0.1$. 


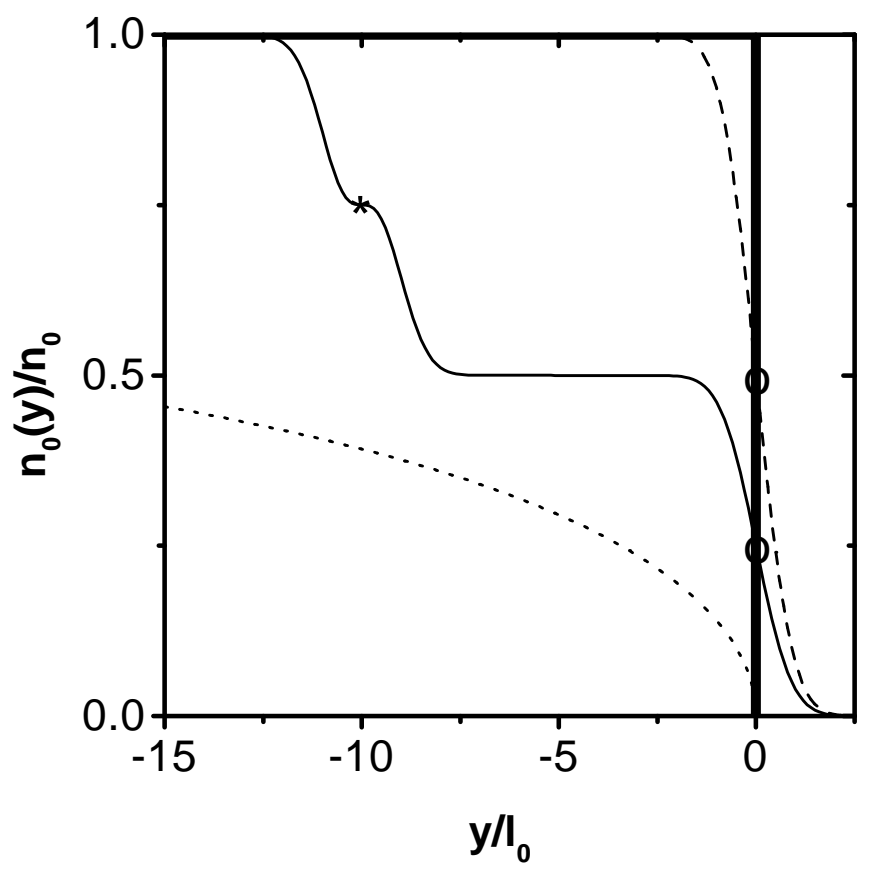

Fig. 1

Balev et al. 


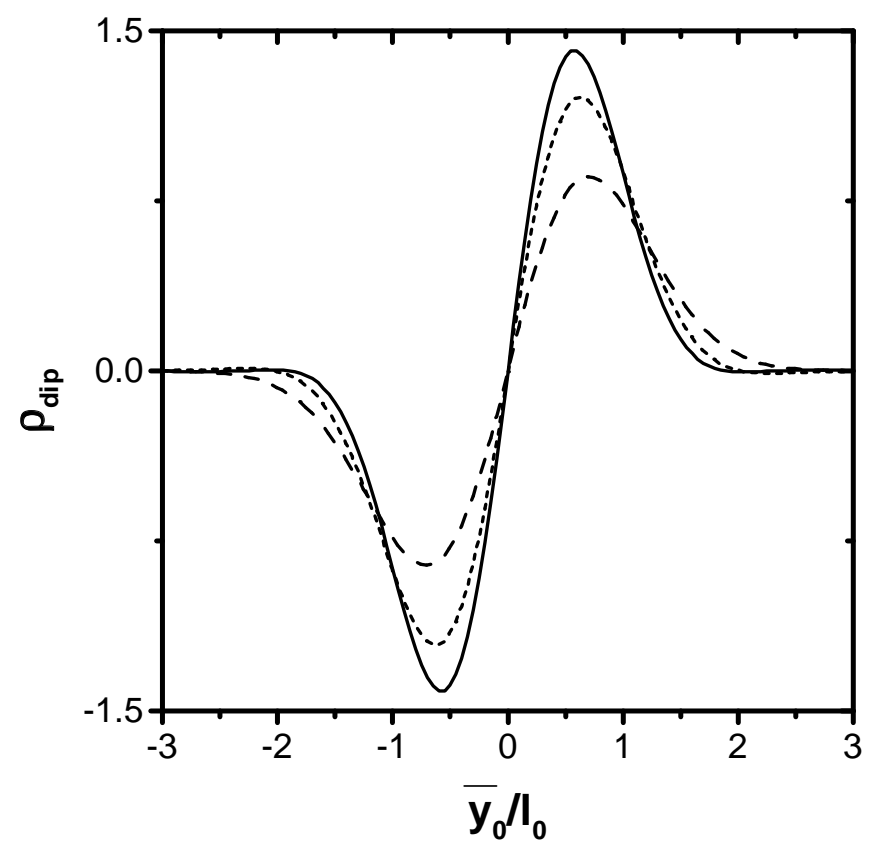

Fig. 2 Balev, et al. 


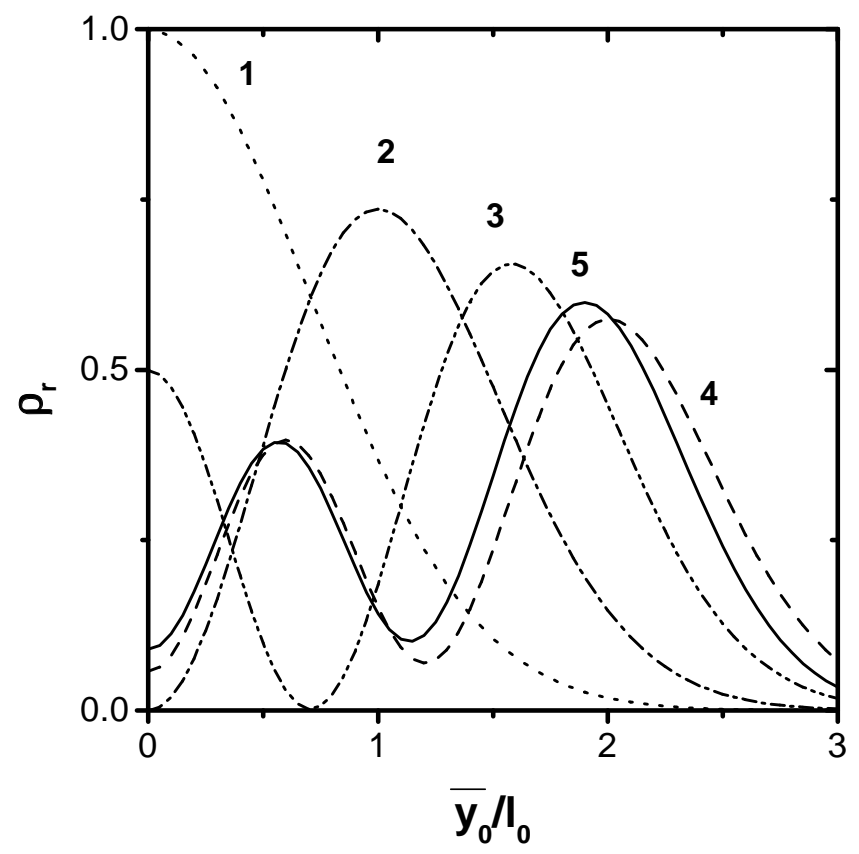

Fig.3 : Balev et al. 


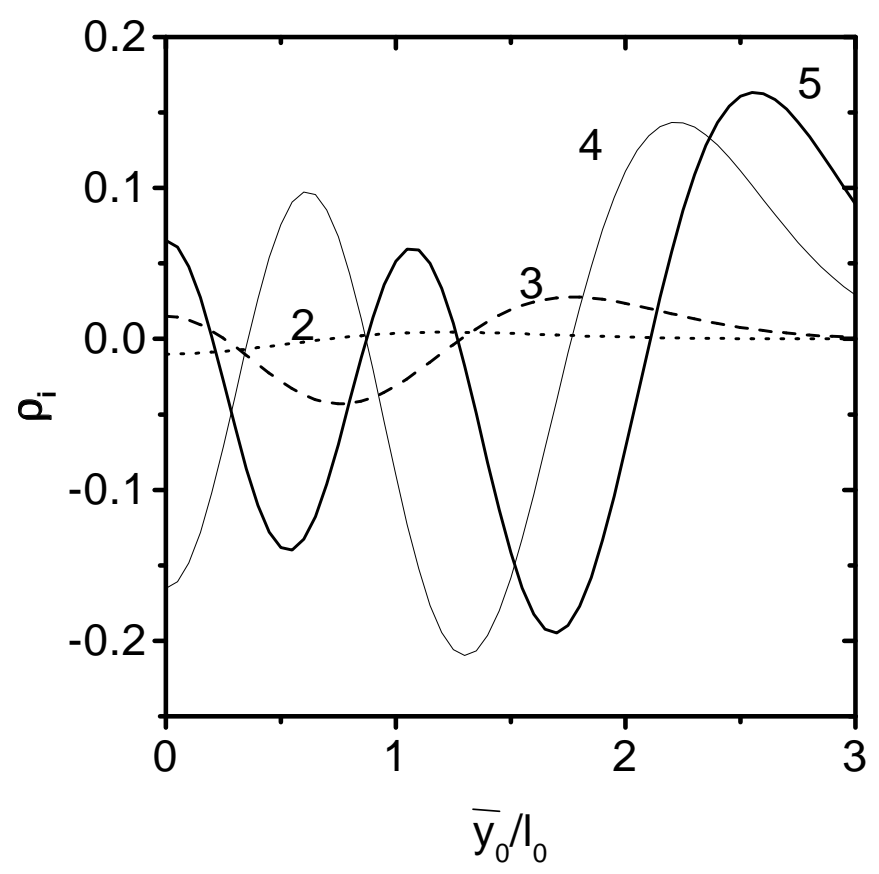

Fig. 4 Balev et al. 


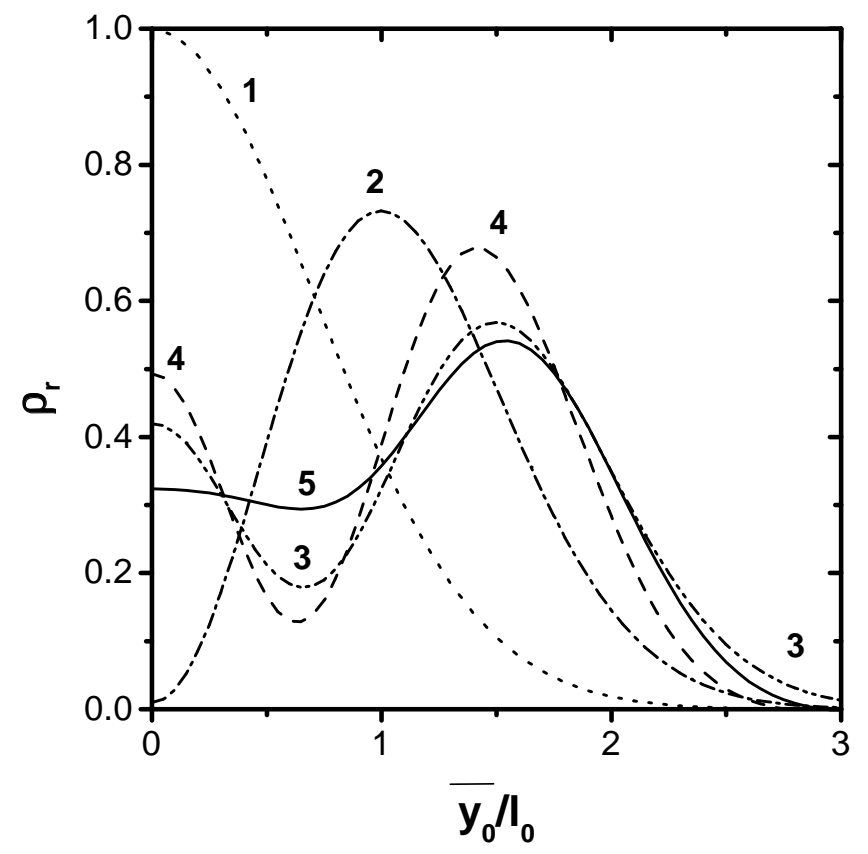

Fig. 5, Balev et al. 


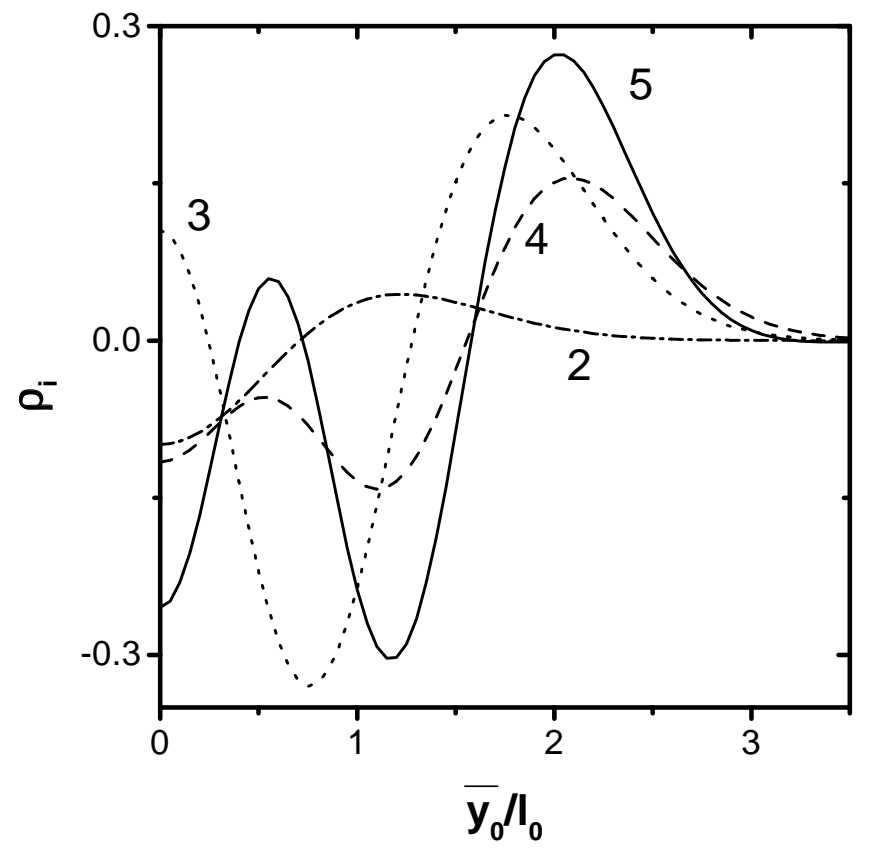

Fig.6, Balev et al. 